\title{
A MULTI-STAGE DISCRETE EVENT SIMULATION APPROACH FOR SCHEDULING OF MAINTENANCE ACTIVITIES IN A SEMICONDUCTOR MANUFACTURING LINE
}

\author{
Wolfgang Scholl \\ Marcin Mosinski \\ Infineon Technologies Dresden \\ Koenigsbruecker Strasse 180 \\ Dresden 01099, GERMANY
}

\author{
Boon Ping Gan \\ Peter Lendermann \\ D-SIMLAB Technologies Pte Ltd \\ 8 Jurong Town Hall Road \#30-04 JTC Summit \\ Singapore 609434, SINGAPORE
}

\author{
Patrick Preuss \\ Daniel Noack \\ D-SIMLAB Technologies GmbH \\ Wiener Platz 6 \\ Dresden 01069, GERMANY
}

\begin{abstract}
Discrete event simulation (DES) has been established as a frequently used decision-support method in semiconductor manufacturing. One of the key application areas is the planning and scheduling of extended (several days) maintenance activities. The first stage of maintenance activity planning is conducted with a transient long-term simulation model with the focus on evaluating the effect of maintenance activity on the expected fab performance. Decisions such as wafer start reduction or adjustment of delivery commitments among affected work centers are made. The second stage of the planning is initiated several days before the start of the maintenance activities, where resource planning and scheduling of the activity is done through assessment of the expected WIP situation forecasted by a high fidelity online simulation model. In this paper, we will explain this simulation-based multi-stage approach for maintenance activity scheduling. The associated benefits and challenges will be presented with an example use case.
\end{abstract}

\section{INTRODUCTION}

Discrete event simulation (DES) has been established as a frequently used decision-support method to address both long/mid-term dynamic planning issues and short term operational execution/production control issues in the Infineon Technologies (Dresden) semiconductor wafer fab facility. Long and mid-term planning requires an abstract simulation model where the model is generated semi-automatically. Full automation is not feasible since data required to portray typical fab behavior over a couple of weeks - such as historical data describing sampling and down behavior and representative process routes describing typical route products flow through the system - is not available from any operational data sources. The key performance indicators (KPIs) of the long/mid-term simulation model focus on the performance trend for a time horizon of one to approximately 15 weeks. On the other hand, addressing short-term operational execution issues requires a fine granularity and complex short-term simulation model. The short-term simulation model needs to be initialized with more detailed data, such as current tool releases, process availabilities (dedication up to individual equipment level), and lot properties. The modeling approach has to be mainly deterministic, with information about expected tool down durations instead of using typical 
values from history analysis and a more detailed sampling modeling using lot attributes instead of historical sampling rates. A short-term simulation model must be generated in a fully automated manner with short computation time, which means $100 \%$ basic data availability as well as data quality are crucial. Any incorrect and missing data must be corrected using an intelligent post processing mechanism as manual basic data correction would be too time-consuming. Abstraction with respect to process routes or dedication information to reduce data complexity is not possible as this would restrict the application of short term simulation. This means that huge data volumes need to be handled within a highly complex model architecture. The key performance indicators (KPIs) of the short-term simulation model focus on categorizing values to different level of criticality, with a time horizon of one to seven days.

One of the key applications of the long/mid-term simulation model is to quantify the effect and side effect on product cycle time and delivery with additional wafer starts or the effect of line incidents. Another application is to evaluate the impact of extended maintenance and engineering schedules to the fab capacity. Typically, a time window of four to six weeks is available to decide for the best. The visibility into the potential impact of different schedules to fab capacity through long/mid-term simulation helps in the decision making of wafer start reduction or delivery commitment adjustment at an early stage. In contrast, a short-term simulation model is used to identify the right point in time (the day of the week) to perform preventive maintenance (PM) activities. This is enabled through the lot arrival forecast for the next seven days using the short term simulation model. PM activities can then be scheduled on the day with low lot arrival rate. This latter application has been deployed in Infineon Technologies (Dresden), where the short term simulation forecast results are made available for daily operational planning, and is accessible by a wide user group through the integration with existing maintenance planning system.

The remainder of the paper is organised as follows: The specific applications and underlying simulation modeling approaches for a yearly PM activity scheduled using long-term and short-term simulation are described in Sections 2 and 3, respectively. A specific use case that illustrates the interdependencies of both approaches and also shows the achievable benefit is discussed in Section 4, followed by conclusions and an outline of future work in Section 5.

\section{LONG-TERM SIMULATION}

\subsection{Application}

At Infineon Dresden discrete-event simulation is integrated into the business process capacity planning and wafer start management. The purpose of long-term simulation is to forecast the fab behavior of the next three to four months, especially key performance indicators such as work-in-progress (WIP), cycle time, moves, wafers out and utilization. This forecast horizon covers the typical response time of the fab to changed input conditions such as wafer starts, uptime and dispatching. Another important application is to quantify both direct effect as well as side-effects of additional wafer starts in a fully loaded fab under consideration of actual dispatching policies.

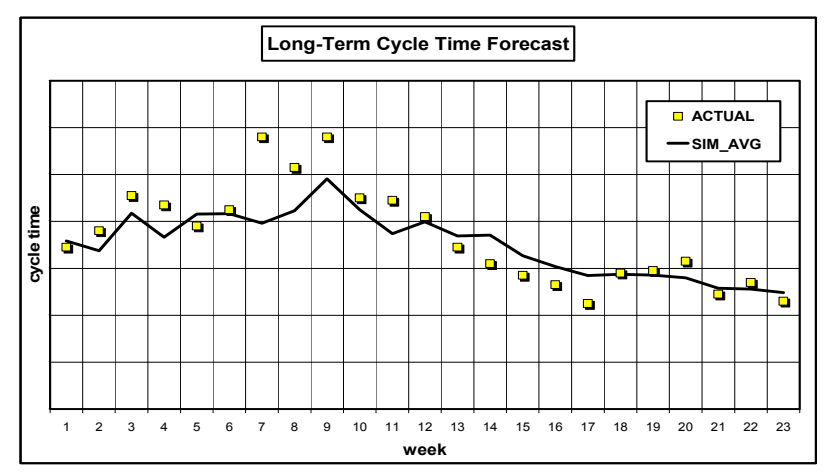

Figure 1: Long-term forecast of fab cycle time after product mix change 


\section{Scholl, Gan, Mosinski, Lendermann, Preuss, and Noack}

Although regular changes in wafer starts are typical for a fab of customer-specific logic products, a long-term forecast of cycle time and WIP on level fab or product group is feasible and useful for both strategic and operational decisions. In Figure 1 it is shown that the long-term simulation forecast can predict the cycle time trend, although various fab constraints have changed during this time.

Another important application is cycle time forecast on work center level. From the point of view of simulation this is a plausibility check and allows inferences to be made about basic data quality. In the production departments it will be used for planning tasks, e.g. correction of tool and process release plans and, of course, for a first early scheduling of several days' engineering and maintenance activities, as described in detail in Section 4 of this paper.

\subsection{Modeling Approach}

The actual line situation in the fab is relevant for a performance forecast between the following week up to a three-month time horizon, so the simulation has to be transient in nature. Long-term simulation is a mainly stochastic and very pragmatic approach.

What are the main attributes of a long-term performance forecast? In addition to usual statistics from history (distribution of down events and time parameters for down events, setup, lot transportation and so on) other typical disturbances such as transport system (AMHS) and IT problems, in the production process have to be considered. These events have a low probability of occurrence, but a high impact on longterm fab performance trends. Both extensive experience and systematic analyses are necessary to find out important "ingredients" of a realistic fab modeling. Selective machines are noticeable with extended downs in addition to their normal down behavior. It has been shown through internal study that a modeling based on average down parameters of MTOL (Mean Time Off Line) and MTTR (Mean Time to Repair) from the last three months' history is not good enough, a separate down distribution has to be used in the simulation. The same is also applied for periodical chamber cleans and bath changes. Due to a lot of stochastic events confidence runs are necessary, but to achieve acceptable computation times the number is limited to ten. This limitation does not have a significant impact on accuracy of the simulation results. A simplified modeling approach is very important to master the huge data volume. The level of simplification is defined in the validation phase and depends on each particular application and on the data availability from data sources. Some simplification features are:

- Use of route representatives based on technological classification criteria

- High level modeling of tool configurations and dispatch rules

- Sampling process modeling based on historical data (rates)

- Modeling of non-productive wafer processing with additional down distributions

- Simplified warm start conditions with consideration of current WIP lot positions only

- Consideration of just the most important dedications within work center (e.g. lithography, sputtering) A model initialization with too much detail, e.g. all currently restricted tool releases, would also handicap the use of the simulation for long-term forecast. Model validation is carried out based on historical production data (WIP, wafer starts, discrete down events and so on) in a step-by-step approach starting on level production area. Once an agreement is reached between simulation and reality, the validation moves on to the next level of details, which is work center level. The typical runtime for the three months simulation forecast of the fab Dresden is around 12 hours. This does not restrict the usability as the forecast horizon smaller than one day is not relevant. It took more than one year to get a realistic fab model for this application.

\section{SHORT-TERM SIMULATION}

\subsection{Application}

In a dynamic manufacturing environment numerous performance disturbances, caused by events such as unscheduled equipment downs, re-entrant flows and large number of product mixes exist (Scholl 2008). 


\section{Scholl, Gan, Mosinski, Lendermann, Preuss, and Noack}

Such disturbances result in lot arrival fluctuations at work center and resulting in performance losses. If, compared to the average arrival rate, the work center lot arrivals are high then work center WIP and cycle time increase. In turn, if the work center lot arrivals are relatively low then work center capacity is lost. In the worst case a preventive maintenance (PM) activity that is not planned properly occurs during a time of high workload rather than during a low WIP period. Badly planned PM occurs today as the PM planning process considers only the urgency of PM, the availability of maintenance resources and maintenance personnel. With the availability of short term simulation that provides work center performance forecast to predict lot arrival and WIP fluctuation on a daily basis, a PM activity can be planned by considering the anticipated workload at the work center as an additional criterion.

\subsection{Modeling Approach}

The essence of the short term simulation model is (Scholl et al. 2010):

- High level of detail to achieve high forecast accuracy

- Model initialization using the current state of fab situation

- High degree of automation to generate the simulation model automatically (and fast)

The short-term simulation model contains a high level of detail, including real routes as maintained in the Manufacturing Execution System (MES), lot attribute dependent sampling, rework, high granularity equipment modeling with consideration of chamber influence, send-ahead modeling, and work center specific dispatching rules including Kanban modeling. Such a detailed model is required to ensure high quality of forecast accuracy to be achieved at work center level for the purpose of operational planning.

Besides having high level of modeling details, a short-term simulation must be initialized with the current state of fab situation. The short-term simulation model does not have to go through a warm-up period as it does not start from an empty fab (Reijers and Aalst 1999). The model initialization contains the current equipment states, including estimated down duration, WIP lot hold and rework status, future lot release with individual lot information and attributes.

A large volume of data is required for the generation of a short-term simulation model with such high granularity. A high degree of automation for model generation is therefore essential. The automatic procedure includes data access, data transformation, model generation and reporting. An automated error identification and error correction is essential to keep the data quality on a high level. It includes the handling of missing lot operations, missing or additional equipment, and incorrect process times.

Our short-term simulation uses a mainly deterministic modeling approach. Stochastic behavior is kept at a minimum and only applies when data with sufficient quality is not available or impossible to obtain. An example is the non-productive equipment state modeling. Preventive maintenance (PM), unscheduled down, and engineering states are the contributing factors to an equipment non-productive state. Information on PM and engineering states are available, and thus stochastic modeling of this element is not essential. But unscheduled down events are not predictable and therefore have to be modeled as stochastic behavior. Another example is lot sampling which also can be modeled as purely stochastic events. However, in our short-term simulation model some elements of lot sampling are modeled using lot attributes, where the lot attributes define the operation that the lot must run (and must not run). This is a mandatory requirement for application of short-term lot arrival forecast in wafer inspection area.

\section{EXAMPLE OF USE CASE}

\subsection{Multi-Stage Approach For Yearly PM Scheduling}

At times when yearly or quarterly preventive maintenance activities (to be executed within a time span of 4-6 weeks and with a duration of 12 or more hours) are required the equipment cannot be used for productive wafer processing. It is therefore important to find suitable time slots when such PM activities can be carried out. The realization of this use case can be done in two steps: First of all the long-term simula- 


\section{Scholl, Gan, Mosinski, Lendermann, Preuss, and Noack}

tion forecast will be evaluated to find a week with a good cycle time of the equipment's work center as shown in Figure 2. For example, work center WC-10 has a good time window in week 6 to week 9 for preventive maintenance activity as these weeks have the lowest cycle time violation forecast.

Having identified the weeks that are most appropriate for preventive maintenance (PM), the shortterm simulation can now be used to find the best time slot during these weeks to execute the PM. The value of the short-term simulation is to give a 7-day of arrival forecast for various work centers across different areas of the fab. These forecasts can be used to derive the expected work load situation at certain areas over the time period. This information helps to further improve the timing of PM activities in regard to the current work center situation. The incumbent PM planning process takes into consideration just two factors: (1) the upcoming PM time window, and (2) the availability of the PM staff, however, it does not consider the work center performance. To address this, a commercial short-term simulation solution $D$ SIMCON Forecaster (D-SIMLAB 2012) has been deployed and integrated with the PM planning system to provide daily work center performance forecasts. PM activities can thus easily be planned to avoid major WIP fluctuations and cycle time increases at the work center.

\begin{tabular}{|c|c|c|c|c|c|c|c|c|c|c|c|c|c|c|c|c|c|c|c|c|c|c|c|}
\hline \multirow[b]{2}{*}{ Workcenter } & \multicolumn{4}{|c|}{ ACTUAL } & \multicolumn{19}{|c|}{ SIMULATION } \\
\hline & $\begin{array}{l}m_{1} \\
x^{\prime} \\
\text { w } \\
3\end{array}$ & 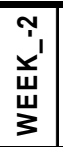 & $\begin{array}{l}T_{1} \\
x^{\prime} \\
w \\
w \\
3\end{array}$ & 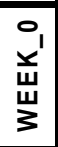 & $\begin{array}{l}\bar{y}^{\prime} \\
\vec{w} \\
\ddot{z}\end{array}$ & $\begin{array}{l}N_{1} \\
\stackrel{x}{ } \\
w \\
3\end{array}$ & $\begin{array}{l}m^{\prime} \\
\underset{w}{w} \\
w\end{array}$ & 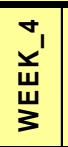 & 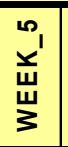 & $\begin{array}{l}\sigma_{1} \\
x^{\prime} \\
\Psi \\
\widetilde{J}\end{array}$ & 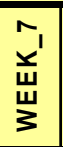 & $\begin{array}{l}\infty \\
\underline{y}^{\prime} \\
\underset{w}{w} \\
\underset{3}{3}\end{array}$ & $\begin{array}{l}\sigma_{1} \\
\stackrel{x}{u} \\
w \\
u\end{array}$ & $\begin{array}{l} \\
x^{\prime} \\
\underset{w}{w} \\
\stackrel{3}{3}\end{array}$ & $\begin{array}{l}F_{1} \\
x^{\prime} \\
w \\
w\end{array}$ & 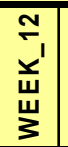 & 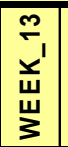 & 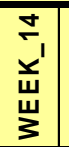 & 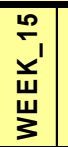 & 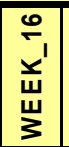 & 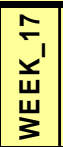 & 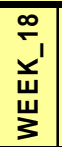 & 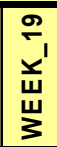 \\
\hline$\overline{W C-1}$ & 0.2 & 0.6 & 1.7 & 0.5 & 2.5 & 1.6 & 2 & 2.4 & 3.6 & 3.1 & 1.5 & 2 & 1.2 & 1.9 & 1.9 & 1.5 & 2.5 & 2.3 & 2 & 2.1 & 2.9 & 2.5 & 2.4 \\
\hline WC-2 & 5.5 & 4.8 & 2.2 & 1.5 & 0.2 & 0.5 & 0.3 & 0.4 & -0.1 & 0.1 & 0.5 & 0.1 & 0 & -0.2 & 0.1 & 0 & $\mid$\begin{tabular}{|l|}
-0.1 \\
\end{tabular} & 0.1 & 0.2 & 0.2 & 0.4 & 0.1 & 0.2 \\
\hline WC-4 & -0.8 & 2.2 & 2.1 & -0.5 & -1.9 & \begin{tabular}{|c|}
-1.4 \\
\end{tabular} & -1.4 & -1.7 & -1.8 & -0.7 & -1.2 & -1.7 & -2 & -1.9 & -2.1 & $\mid-2.1$ & -2.2 & -2.2 & -2.1 & $\mid-2.1$ & -2 & -2.2 & -2 \\
\hline WC-5 & 0.6 & -2.2 & -2.3 & $\begin{array}{l}-1.6 \\
\end{array}$ & -2.1 & -2.1 & -1.7 & \begin{tabular}{|c|}
-1.5 \\
\end{tabular} & -2 & -1.9 & \begin{tabular}{|c|}
-1.9 \\
\end{tabular} & $\begin{array}{ll}-1.7 \\
\end{array}$ & -1.8 & -2 & -1.8 & -1.8 & \begin{tabular}{|l|}
-1.9 \\
\end{tabular} & -2 & -2 & $\mid-1.8$ & \begin{tabular}{|l|}
-1.4 \\
\end{tabular} & -1.8 & -1.7 \\
\hline WC-6 & 0.3 & 1.1 & 2.2 & 1.4 & 0.8 & 0.5 & 1.9 & 1.6 & 0.4 & -0.1 & 0.1 & 0 & 0.1 & \begin{tabular}{|c|}
-0.1 \\
\end{tabular} & 0.1 & \begin{tabular}{l|}
-0.2 \\
\end{tabular} & \begin{tabular}{|l|}
-0.3 \\
\end{tabular} & 0.1 & -0.3 & \begin{tabular}{|l|}
0.3 \\
\end{tabular} & \begin{tabular}{|l|}
0.2 \\
\end{tabular} & -0.1 & 0.2 \\
\hline WC-7 & 5.1 & 0.7 & 2.1 & 0.6 & 4.2 & 2.7 & 0.5 & 0.6 & 0.7 & 0.5 & -0.6 & 0.2 & \begin{tabular}{l|l|}
-0.6 \\
\end{tabular} & -0.7 & $\mid-0.5$ & -0.6 & \begin{tabular}{|l|}
-0.6 \\
\end{tabular} & \begin{tabular}{|l|}
-0.7 \\
\end{tabular} & -0.6 & \begin{tabular}{|l|}
-0.7 \\
\end{tabular} & \begin{tabular}{|l|}
-0.8 \\
\end{tabular} & -0.8 & -0.8 \\
\hline WC-8 & 10.4 & 69.3 & 68.4 & 30.2 & 8.6 & 6.4 & 3.3 & 2.6 & 1.5 & 2 & 2.6 & 1.9 & 3.3 & 3.4 & 1.6 & 2.3 & \begin{tabular}{|l|}
1.7 \\
\end{tabular} & \begin{tabular}{|l|}
1.7 \\
\end{tabular} & 2.7 & \begin{tabular}{|l|}
0.7 \\
\end{tabular} & 3 & 4.5 & 2.7 \\
\hline WC-9 & 5.8 & 5.1 & 1.8 & 9.8 & 2.7 & 4.1 & 2.4 & 2.7 & 2 & $2 n$ & $0 . \overline{1}$ & $\tilde{2.0}$ & 13 & 5.7 & 2.5 & 3 & 2.8 & 2.4 & 3.8 & 0.7 & 4 & 6.3 & 3.7 \\
\hline WC-10 & 147 & 101 & 27.2 & 15.3 & 14.9 & 2.5 & 15.4 & 26.4 & 14. & 0.3 & -0.2 & 0 & 0.11 & 1 & 0.5 & 1.2 & \begin{tabular}{|l|}
0.2 \\
\end{tabular} & 0.2 & 0.8 & 0 & \begin{tabular}{|l|}
-0.8 \\
\end{tabular} & -0.7 & -0.5 \\
\hline WC-11 & -0.8 & -0.6 & 2.7 & -0.4 & -0.9 & -1.5 & -0.7 & -1.2 & -1.6 & 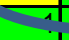 & 13 & -12 & 7.3 & -1.4 & \begin{tabular}{|l|}
-1.2 \\
\end{tabular} & \begin{tabular}{|l|}
-1.2 \\
\end{tabular} & \begin{tabular}{|l|}
-1.5 \\
\end{tabular} & \begin{tabular}{|l|}
-1.1 \\
\end{tabular} & \begin{tabular}{|c|}
-1.5 \\
\end{tabular} & \begin{tabular}{|l|}
-1.4 \\
\end{tabular} & \begin{tabular}{|l|}
-1.2 \\
\end{tabular} & \begin{tabular}{|c|}
-1.7 \\
\end{tabular} & -1.5 \\
\hline
\end{tabular}

Figure 2: Long-Term Simulation - Target cycle time violation in hours per week and work center

One of the essential element in integrating D-SIMCON Forecaster and the PM planning system is to provide an easy way for maintenance department specialist to access and understand the forecast results. They have to be presented in a precise and user friendly manner so that the main focus of the department is not to focus on how to interpret the report, but more on how to make use of it. This is achieved through a 7-day simulation forecast in some abstracted overview chart in shape and colour of a traffic light for each forecast day, whereby the colours indicate whether or not a PM activity on the particular day is recommended. Each work center gets assigned a particular readiness level to perform PM, based on the wafers arrival forecast. One example of classification would be based on a "tact rate" (frequency) or thresholds defined by the production department.

In our approach, the readiness level is denoted by 3 colours: Green indicates bringing down an equipment of the work center for PM would not cause any major WIP issue, yellow indicates this would have minor effect on WIP, and red indicates this would cause a significant bottleneck situation.

For a work center where PM can be performed (green or yellow), the user needs to identify the equipment to be brought down for PM. The mapping of work centers to equipment is therefore crucial. Table 1 gives the specification to visualize the equipment readiness for PM. The table shows the equipment PM readiness for today " $+0 \mathrm{~d}$ ", for tomorrow " $+1 \mathrm{~d}$ " and all other days of the week. For example, the best time to perform PM for ASH001_01 is to postpone it to the fifth or sixth days. Within the first 3 days the equipment is not suitable to execute any PM. 


\begin{tabular}{|c|c|c|c|c|c|c|c|c|c|c|c|c|c|}
\hline \multicolumn{6}{|c|}{ Available PM Planning Information } & \multicolumn{7}{|c|}{ New Forecast Information } & \multirow[b]{2}{*}{ Duration } \\
\hline Equipment & Id & Start Date & Prio & Staff & .. & $+0 \mathrm{~d}$ & $+1 \mathrm{~d}$ & $+2 \mathrm{~d}$ & $+3 \mathrm{~d}$ & $+4 \mathrm{~d}$ & $+5 \mathrm{~d}$ & $+6 \mathrm{~d}$ & \\
\hline ASH001_01 & 1 & $\begin{array}{l}23.4 .2012 \\
00: 00: 00\end{array}$ & 3 & Mrs. B & & & & & & & & & $8 \mathrm{~h}$ \\
\hline ETC002_02 & 2 & $\begin{array}{l}23.4 .2012 \\
01: 23: 44\end{array}$ & 1 & Mrs. B & & & & & & & & & $1 \mathrm{~h}$ \\
\hline FOT003_03 & 3 & $\begin{array}{l}23.4 .2012 \\
14: 23: 44\end{array}$ & 5 & Mr. Q & & & & & & & & & $48 \mathrm{~h}$ \\
\hline SPU005_05 & 4 & $\begin{array}{l}19.5 .2012 \\
14: 23: 44\end{array}$ & 3 & Mr. Z & & & & & & & & & $24 \mathrm{~h}$ \\
\hline
\end{tabular}

Table 1: Illustration PM Planning Tool with Forecast Information

As a first step towards establishing short-term simulation in the production departments as use case, for each focus department an individually designed intranet web page has been created, containing the arrival forecast for the department work center as well as the abstracted visualization of the PM forecast information. This approach allows focusing on selected work centers while talking to the production areas over few weeks to validate the forecasts, modify thresholds used for the PM decision support chart and deepen the acceptance of the simulation forecasts. An example of this intranet visualization can be found in Figure 3 .

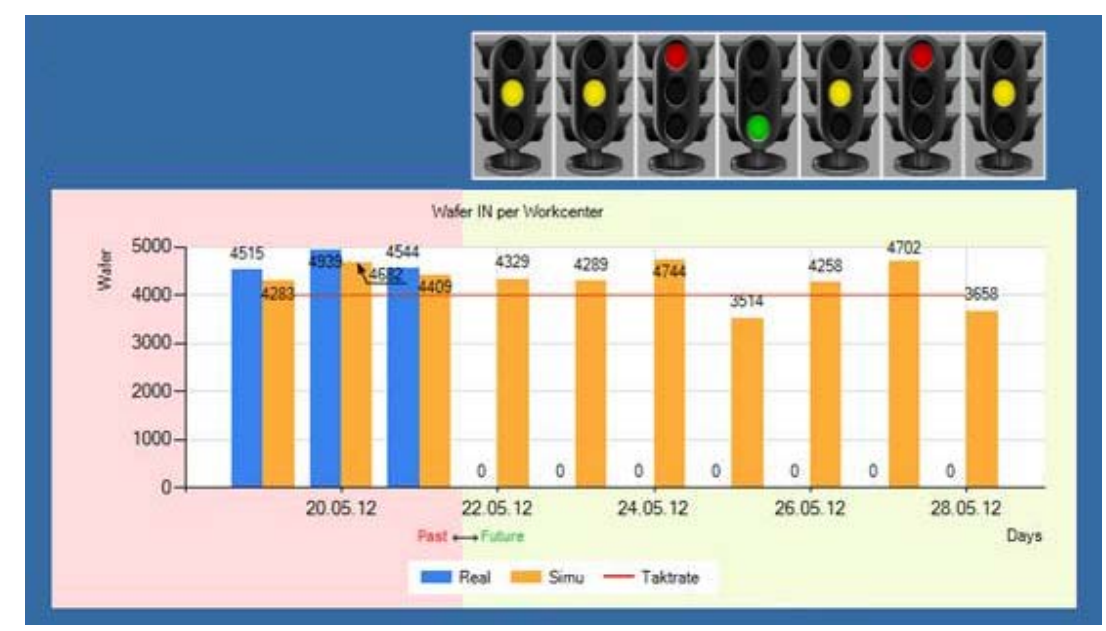

Figure 3: Department specific simulation forecast, including PM-planning decision support

Figure 4 shows a screen capture of the integrated PM planning system and short-term simulation forecast. This solution is accessible fab-wide and allows different departments to make use of the simulation forecast results to identify good timings to carry out upcoming PM activities. To increase the ability to shift a PM to the suggested period and decrease the constraints which occur due to the availability of the PMoperators, this system is going to be integrated with an existing solver technology that creates the work schedule for PM personnel. Integrating the solution provides a mean for the solver to allocate the right PM-operators at the right time, to avoid a planned PM without PM personnel actually being available. 


\begin{tabular}{|c|c|c|c|c|c|c|c|c|c|c|}
\hline \multicolumn{11}{|c|}{ Edit View Refresh ? } \\
\hline \multicolumn{11}{|c|}{ Drag a column header here to group by that column. } \\
\hline \multicolumn{10}{|c|}{ PPPMPLan } & \multirow{2}{*}{$\begin{array}{l}\text { FORECAST HORIZON } \\
\text { (EXAMPLE) }\end{array}$} \\
\hline \multirow{2}{*}{, } & Equipment $\nabla$ & PROZESS & $\nabla$ Di. $\nabla$ & Eckstart $\nabla$ & Dauer gepl. & PlanKW $\nabla$ & Auftrag $\nabla$ & GRUNDLANG & $\nabla$ Grund $\nabla$ & \\
\hline & ETC031 & 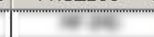 & ! & 09.04 .2012 & 4 & 152012 & 101915512 & halbjährliche Wartung & HALB & \\
\hline 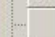 & ETC024 & $2+x+4$ & & 10.04 .2012 & 72 & 152012 & 101919607 & jährliche Wartung & JÄHR & \\
\hline- & $\overline{\text { ETC010 }}$ & 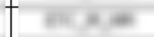 & & 11.04 .2012 & 50 & 152012 & 101919605 & jährliche PM + BGVA3 & JÄHR & \\
\hline - & $\overline{\text { ETC031 }}$ & antary & & 11.04 .2012 & 4 & 152012 & \begin{tabular}{|l|}
101915530 \\
\end{tabular} & jährliche Wartung & JÄHR & \\
\hline - & $\overline{\text { ETC016 }}$ & 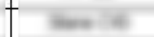 & & 12.04 .2012 & 48 & 152012 & \begin{tabular}{|l|}
101918563 \\
\end{tabular} & jährliche Wartung & JÄHR & \\
\hline+ & ETC031 & enes & & 15.04 .2012 & 16 & 162012 & 101919378 & jährliche Wartung & JÄHR & \\
\hline+ & $\overline{\mathrm{ETC} C 22}$ & - & & 17.04 .2012 & 26 & 162012 & 101921869 & jährliche Wartung + BGVA3 & JÄHR & \\
\hline 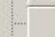 & $\overline{\mathrm{ETC} 031}$ & 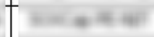 & & 28.04 .2012 & 16 & 172012 & 101923315 & jährliche Wartung & JäHR & \\
\hline- & $\overline{\text { SPU001 }}$ & exing & & 01.05 .2012 & 72 & 182012 & \begin{tabular}{|l|}
101926722 \\
\end{tabular} & jährliche Wartung & JÄHR & \\
\hline- & $\overline{\text { ETCO31 }}$ & ater & & 02.05 .2012 & 4 & 182012 & 101922152 & halbjährliche Wartung & HALB & \\
\hline- & $\overline{\text { SPU002 }}$ & 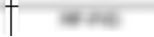 & & 02.05 .2012 & 72 & 182012 & 101926019 & jährliche Wartung & JÄHR & \\
\hline
\end{tabular}

Figure 4: Equipment maintenance application with integrated PM decision support on the right

\subsection{Effect On Fab Performance}

Figure 5 below illustrates the benefit that was achieved through the use of short term simulation for preventive maintenance (PM) planning in wafer inspection area. Before 24 May PM activities were planned without using short-term simulation forecast. As can be seen, the WIP level increases in all cases, e.g. by 470 wafers between 18 April and 20 April and by 400 wafers between 24 April and 26 April. As the PM was executed with consideration of forecasted lots arrival after 24 May the PM activities do not result in WIP level increases. For the PM between 24 May and 26 May the WIP level actually decreases by 280 wafers, for the PM between 30 May to 1 June the WIP level decreases by 500 wafers, and for the PM between 5 June and 7 June, the WIP level decreases by 700 wafers. This was observed as the PM was planned on the days when lot arrival at the respective work centers was low.

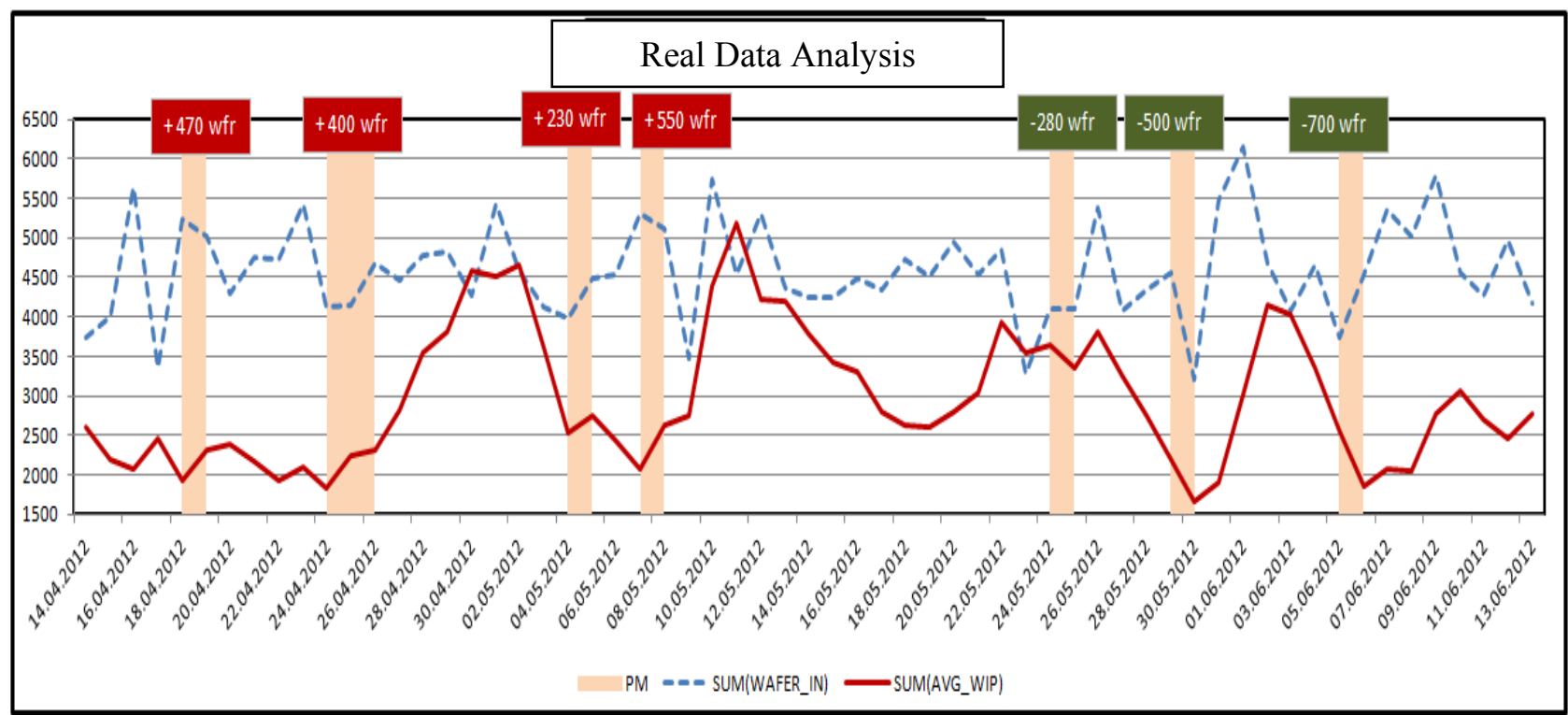

Planning of Weekly PM without Lot Arrival Forecast

and with Short-Term Simulation

Figure 5: Equipment maintenance application with integrated PM decision support on the right 


\section{CONCLUSIONS AND FUTURE WORKS}

In this paper we have described an approach of combining both long/mid-term simulation and short-term simulation for PM planning in a semiconductor wafer fab environment. Using this approach, PM planning takes into account not only time windows and resource availability but also anticipated lot arrivals at the respective work centers. The long/mid-term simulation model is used to identify the time period in which PM can be conducted, based on assessment of cycle time impact of the extended PM activities. Upon identifying the week for PM, the short-term simulation model is then used to identify the day within the week that the PM can be performed. This solution is integrated with the Infineon PM planning system, accessible anywhere within the fab. It provides a visual means (traffic light approach) for PM planning specialists to identify the appropriate time to carry out PM activities. This solution has been deployed for a few key work centers, and is in the process of being extended to other work centers across the entire fab. Moving on, further extension of the solution to harmonize with resource planning (PM-operator) will be done to maximize the benefit of simulation-driven PM planning.

Another extension would be to use an additional dynamic tact rate for each of the forecasts instead of a static one for the creation of the PM planning chart. For example, a requirement of having at least one "green light" indicating a time for a PM could be imposed in case a PM is due and must be started during the next week. The simulation can then be rerun with different PM scenarios during the week where the PM is carried out at different days with low arrival situations to find the optimal PM timing for a given objective like cycle time reduction for the work center or to look at other criteria which could improve the global key performance indicators of the fab.

Besides further extending the application of DES, it is crucial to provide continuous maintenance activities to ensure accuracy and benefit of the short-term simulation. These activities comprise continuous basic data maintenance, automatic model verification and validation, feedback from production areas regarding equipment capability updates, lot flow changes, expected equipment downs as well as continuous forecast quality measurement and on-going simulation model improvement. These elements have been visualized in Figure 6.

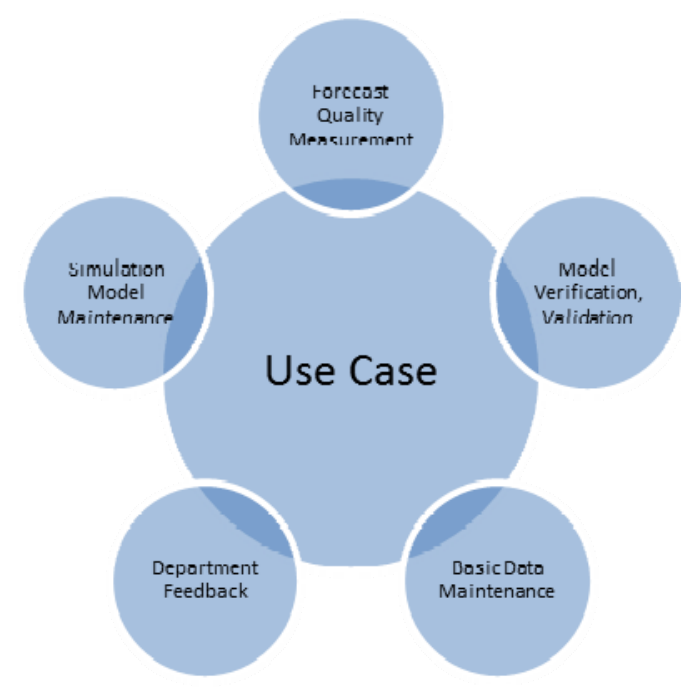

Figure 6: Requirement to ensure a constant accuracy and benefit of simulation-based use 
Reijers, H.A., W.M.P. van der Aalst. 1999. "Short-term simulation: bridging the gap between operational control and strategic decision making", In Proceedings of the IASTED International Conference Modeling and Simulation (MS '99).

Scholl, W. 2008. "Coping with typical unpredictable incidents in a logic fab". In Proceedings of the 2008 Winter Simulation Conference edited by S. J. Mason, R. R. Hill, L. Mönch, O. Rose, T. Jefferson, and J. W. Fowler, 2030-2034. Piscataway, New Jersey: Institute of Electrical and Electronics Engineers, Inc.

Scholl, W., B.P. Gan, D. Noack, P. Preuss, M.L. Peh, P. Lendermann, and O. Rose. 2010. "Towards Realization of a High-Fidelity Simulation Model for Short-Term Horizon Forecasting in Wafer Fabrication Facilities." In Proceedings of the 2010 Winter Simulation Conference, edited by B. Johansson, S. Jain, J. Montoya-Torres, J. Hugan, and E. Yücesan, 2563-2574. Piscataway, New Jersey: Institute of Electrical and Electronics Engineers, Inc.

D-SIMLAB. 2012. "D-SIMCON - An Overview”. http://www.d-simlab.com/dsimcon.htm. Accessed Jun. 1,2012

\section{AUTHOR BIOGRAPHIES}

WOLFGANG SCHOLL works as a Senior Staff Expert for modeling and simulation for Infineon Technologies in Dresden (Germany). He has studied physics at the Technical University of Chemnitz (Germany) and graduated in solid-state physics in 1984. From 1984 to 1995 he worked as a process engineer for ZMD in Dresden. In 1996 he joined Infineon Technologies (former SIMEC) and worked on the field of capacity planning. Since 2003 he is responsible for fab simulation. His email address is wolfgang.scholl@infineon.com.

BOON PING GAN is the CTO of D-SIMLAB Technologies in Singapore. He has been involved in simulation technology application and development since 1995, with primary focus on developing parallel and distributed simulation technology for complex systems such as semiconductor manufacturing and aviation spare inventory management. He is now responsible for managing the technology and product development at D-SIMLAB as well as execution of projects in the semiconductor and engineering domains. He holds a Master of Applied Science degree, specializing in Computer Engineering. His email address is boonping@d-simlab.com.

MARCIN MOSINSKI is a PhD student at Munich University of the Federal Armed Forces. He is a member of the scientific staff of Professor Oliver Rose at the Chair of Modeling and Simulation. He works as a Simulation Expert for a Short Term Simulation for Infineon Technologies in Dresden. He received his M.S. degree in computer science from Dresden University of Technology. His research interests include simulative and analytical forecasting of complex problems in manufacturing facilities and the statistical data analysis. His email address is marcin.mosinski@infineon.com.

PETER LENDERMANN is the Co-Founder and CEO of D-SIMLAB Technologies, a Singapore-based company providing simulation-based decision support solutions and services to Aerospace, Semiconductor Manufacturing and other asset-intensive industries. Prior to this he worked at the Singapore Institute of Manufacturing Technology where he led the simulation-related research activities until spinning them off into D-SIMLAB Technologies. He has been engaged in the simulation community since the early 1990's. Peter holds a PhD in Applied High-Energy Physics from Humboldt University in Berlin (Germany) and an MBA in International Economics and Management from SDA Bocconi in Milan (Italy). He is also an Adjunct Associate Professor at the Department of Industrial and Systems Engineering at the National University of Singapore. His email address is peter@d-simlab.com. 
PATRICK PREUSS is a Software Engineer and the Deputy Manager Germany Operations of DSIMLAB Technologies (Germany). He has been working in the development of simulation-based applications for Airbus, German Aerospace Centre and Infineon with focus on heuristic optimization methods since 2005. Patrick holds a M.S. degree in computer science from Dresden University of Technology. His email address is patrick@d-simlab.com.

DANIEL NOACK is a Senior Simulation Specialist at D-SIMLAB Technologies. His focus is on modeling, simulation-based optimization, and forecasting. He is a $\mathrm{PhD}$ student at Munich University of the Federal Armed Forces. He is a member of the scientific group of Prof. Dr. Oliver Rose at the Chair of Modeling and Simulation. He received his M.S. degree in computer science from Dresden University of Technology. His email address is daniel@d-simlab.com. 